\title{
Personalisation in Cyber Physical and Social Systems: the Case of Recommendations in Cultural Heritage Spaces
}

\author{
Yannick Naudet*, Bereket Abera Yilma*† and Hervé Panetto ${ }^{\dagger}$ \\ ${ }^{*}$ Luxembourg Institute of Science and Technology (LIST) \\ †Université de Lorraine, CNRS, CRAN \\ \{yannick.naudet, bereket.yilma\}@list.lu, herve.panetto@univ-lorraine.fr
}

\begin{abstract}
Providing recommendations to users evolving in physical spaces has been a research trend since a few years now, especially with the rise of recommending engines dedicated to cultural heritage spaces like museums or tourism. The evolution of our civilisation towards more and more pervasiveness of technology in our environment leads to define the spaces in which human-being evolve as Cyber-Physical-Social Systems (CPSS), in which humans cohabit with sensors, actuators, IT devices or robots, all of these entities interacting together. In this position paper, we formalised the recommendation problem in physical spaces from the general perspective of CPSS, introducing its multiple dimensions.
\end{abstract}

\section{INTRODUCTION}

The evolution of our civilisation towards more and more pervasiveness of technology in our environment leads to define the spaces in which human-being evolve as Cyber-PhysicalSocial Systems (CPSS): systems (or environments) in which humans cohabit with sensors, actuators, IT devices or robots, all of these entities being connected and interacting together.

The emerging research fields of Smart City, Smart Enterprise, Smart Transport or Smart Health, etc., which are pushed by societal challenges identified by the European Union (see e.g. the Cyber-Physical European Roadmap and Strategy [1], are typically grounded in CPSS environments (see e.g. [2] for CPSS and Smart City). The concept of CPSS is an extension of Cyber-Physical System (CPS), highlighting the human factors which were more or less forgotten in CPS. In short, CPS connect the physical world to the cyber world, while CPSS additionally connects the social world [3]. Although the term is not often used, Smart Cultural Heritage refers also to a transformation of cultural heritage spaces into CPSS. Indeed, culture is accessible in the physical world and in the cyber world, while being social by essence. The next trend is to mix everything.

The human dimension is particularly important in smart cultural heritage spaces (as it should also be in any other CPSS), putting automated (i.e. supported by Information Technologies, aka IT) personalisation as a central element. However as a central element, it has to deal with the multiple elements and dimensions of the CPSS. This is what this paper is about. We first introduce the common view on IT personalisation and then develop a new approach adapted to CPSS, focusing on

978-1-5386-8225-818\$31.00 (c) 2018 IEEE the case of personalised recommendations in physical cultural heritage spaces.

The research field of recommender systems is rather old now and rich of a lot of works. Recommender systems have been historically defined in different manners and from different perspectives, however, to the best of our knowledge, no formalisation has been provided so far from the general perspective of CPSS.

\section{PERSONALISATION IN INFORMATION TECHNOLOGIES: THE CLASSICAL CASE}

From a general perspective, personalisation in IT is about adapting IT services or applications to the preferences or interests of individuals. It is well known now that having information about the user is not necessarily enough to provide personalised services, especially because interests and preferences are often context-dependent. Context plays an important role, and as part of this context, the application context plays a particularly interesting role. One of its dimension is often not emphasized, because it is implicitly taken into account when the IT application is designed.

Indeed, the final objective of the service an application offers is rarely only the satisfaction of the end-user. Even in the case of a personalised service, personalisation is simply a mean to reach this final objective. The world of recommender systems, which has its roots in the e-commerce domain, illustrates easily the previous statement: the final objective of offering personalized recommendations is to sell something to the end-user, hence the term "consumption" quite often used. Hopefully, making money is not always the ultimate goal, as personalized recommendations can be used for other purposes like, e.g., education or culture.

Satisfying the main purpose or final objective of a personalized service implies making compromises on user interests, or exploiting them in such a way it serves the objective as this is typically the case in e-commerce. This statement implies that personalisation is always about finding the best compromise between user interests / preferences and the application objective, exactly like it has always been between merchants and clients, taking into account any possible influencing contextual element. A personalization problem $P_{u}$ for a user $u$ is a function of this user $u$, an application $a$ and a context $c$ :

$$
P_{u}^{(\text {classic })}=f(u, a, c),
$$


where the application $a$ implements the specific personalisation objective pursued and the context $c$ is a set of elements related to $a$ or $u$ but external to them (i.e. from their environment), which can have an influence on the personalisation objective (e.g. rules regarding the kinds of users, regulations, etc.). A user is by definition someone that uses an application a, i.e. the set of users $U$ verifies $\forall u \in U$, uses $(u, a)$.

Applied to personalised recommendation, equation (1) becomes:

$$
P R_{u}^{(\text {classic })}=f(u, I, a, c),
$$

where $I$ is a set items to recommend, the application $a$ providing personalized recommendations, i.e. a filtered set of the items in $I$ to the user $u$, knowing the influencing context $c$.

\section{Cyber Physical Social Systems AND PERSONALISATION}

\section{A. Background on CPSS}

A common understanding is that Cyber-Physical-Social System (CPSS) is a system comprising three intertwining subsystems (i) The human-based system which refer to the social system containing human actors and their interconnected devices/agents and/or social platforms providing human-based services, (ii) The software-based system that refer to the cyber world providing software-based services including the underlying infrastructures and platforms, either on-premise or in the Cloud and (iii) The thing-based systems referring to the physical world that includes sensors, actuators, gateways and the underlying infrastructures [4].

Although the above definition is commonly used, there are other views, which we do not detail here. The development of CPSS, where social refers to the human aspects is still at it's early stage, especially because it often faces inevitable system instabilities, mainly due to the fact that human's actions and behaviour are the result of individual preferences, cognition, motivation and other natural or/and environmental factors. Moreover, each person is unique and might not follow system's rules that are not aligned with his way of thinking, convictions, etc. Indeed, human behaviour is driven by complex phenomena that we do not fully understand and are still difficult to predict and manage compared to machines and software. All of these aspects characterise CPSS, and were not considered much in research about CPS. We think Personalization might be a solution to handle human behaviour complexity in CPSS; personalization of IT services and devices, or more generally of Human-Machine Interaction. However this makes it also a complex problem for personalisation, which we detail and discuss in the following sections.

\section{B. Personalisation in CPSS}

In CPSS, users of personalized services evolve in an environment inducing constraints making the personalisation problem more complex. Indeed the user evolves in a physical space, with other persons. His behaviour is constrained by the first, and influences the latter, while at the same time he is influenced by the two. Additionally, the physical space has itself a given purpose, calling for expected specific behaviours of people inside. This particular context leads to formulate the personalisation problem differently, as a function of the user $u$, the physical space $s$, the crowd of other persons in the physical space $c r$, the application $a$ and the context $c$, the latter being feed typically by sensors' observations:

$$
P_{u}^{(C P S S)}=f(u, s, c r, a, c)
$$

Applied to personalised recommendation, equation (3) becomes:

$$
P R_{u}^{(C P S S)}=f(u, I, s, c r, a, c) .
$$

Indeed, the complexity of managing personalisation in CPSS has been introduced in [5], with the notion of Personalized Crowd Systems. Given the definition of a Crowd System, a CPSS is equivalent to a Physical Crowd System, and is therefore sensible to perturbations caused by the crowd behaviour. This means that all ends-up to trying to keep the system (the CPSS) equilibrium by fulfilling its objectives and respecting its constraints, while fulfilling also the objectives and interests of the humans inside, and this comes with a certain level of control applied by the system on the users (see [5] for more details). Finally, we propose the following definition for personalised recommendation:

Definition 1: Personalised recommendation is the problem of finding the subset $\operatorname{Irec}_{u} \subset I$ that an application $a$ can suggest to a user $u$, under the set of constraints $C O=C O_{u} \cup$ $C O_{s} \cup C O_{c r} \cup C O_{c}$ induced by $u$, the space $s$ in which he evolves, the crowd $c r$ to which he belongs and the influencing context $c$, and fulfilling at best the set of objectives $O=O_{u} \cup$ $O_{s} \cup O_{a}$ linked to $u, s$ and $a$.

Indeed, personalisation has to take into account all the constraints from the environment where it happens. Then, its objectives are mainly those of the user (e.g. matching needs or preferences), but also of the application (i.e. of the personalisation provider). As the space can be organised or build specifically for a given purpose, this objective still needs to be considered. However the crowd and the context provide only constraints without bringing additional objectives. Intuitively, if a crowd can have objectives, it is out of the scope of bringing personalisation targeting an individual.

\section{THE EFFECT OF PERSONALISATION}

Bringing personalised IT services to an individual has an impact on the CPSS in which this individual evolves, because it somehow drives or limits her actions. The potential impact of personalisation is more evident in physical spaces. Adaptations of the physical spaces, personalised guidance or personalised recommendation of multimedia content have all an impact on the way the user interacts with the physical space, modifying his normal behaviour and consequently impacting the behaviour of other persons in that space.

In virtual spaces where the classical personalisation process occur, the problem as defined by equation (1), although reflecting the common approach, brings a reductionist view. There are chances that collaborative filtering approaches to recommendation, proposing e.g. items other users have already seen, tend to drive users to the same items. In the multimedia domain, this issue of the long tail of not recommended resources is well known [6] and has in particular given raise of works on the diversity of recommendations. Hence the 
behaviour of the user interacting with the personalised service potentially impacts the behaviour of other users, like in the physical space. Investigating further on the same example, the long tail can have an impact on the system where personalisation is offered (here, e.g., an e-commerce site): the distribution of sold resources can be important for the financial viability.

With those examples, we illustrated that even in virtual spaces, the variables of the personalisation problem are actually the same as defined in equation (3). The inherent complexity of CPSS is indeed also present in virtual spaces, which makes that the approach we propose for CPSS can be adopted in any cases.

However, the personalisation strategy formalised by the function $f$ will change according to the context and the kind of personalisation. More generally, the CPSS also impacts personalisation. The following section discusses this impact in the recommendation case.

\section{THE IMPACT OF THE SYSTEM ON RECOMMENDATION}

The objectives and constraints attached to the system have a direct impact on the way recommendations are computed. In e-commerce or multimedia recommendation, the user chooses one item in the list of recommended ones. This is the goal of the system. In a physical space, recommendations are tight to the user location. The goal then becomes to propose the right items at the right location. Context-aware recommendations will consider the right moment, the right activity, etc... However in the usual use cases, this is a one-time goal for the system: upon a recommendation, the user chooses one item, and the next recommendation is almost independent from the one before as it answers to a different need. When the system's goal is as complex as maximising a visit experience, like in museums or tourism tours, recommendations are no more independent because the objective targets no more one recommendation, but rather a set of recommendations provided during a known period of time.

Personalised recommendations in a museum reach the system's goal not only when each recommendation matches the user expectations, but rather when the set of recommendations offered during the complete visit constitute a coherent whole. Recommendations consists no more in a set of items, but in a set of coherent sequences of items, which can also be named paths. Independently from the way recommendations are presented, as a sequence or as the next step to follow, the resulting actual path followed by the user has to be coherent. This coherency is subject to space and time limits (e.g. not a too large distance between consecutive items or total scheduled visit time matching the museum or the user constraints). Depending on the museum's or exhibition's goals, diversity or focus of the sequence, or generated surprise can be objectives to fulfil. But before all, it needs to be consistent with a given narrative.

\section{PERSonalised ReCOMmendations in A Museum}

In this section we develop our approach to the case of personalized recommendations in a museum, which is, together with the tourism domain (e.g. city visits) the most common instantiation of personalisation in cultural heritage spaces.

\section{A. The recommendation problem in a museum}

The recommendation problem in a museum can be defined as follows. Let $E$, be the set of main entities of interest which are part of the museum system. This set is necessary time dependent, as it is considered at the moment recommendation is to be computed and all its elements may vary each time it is done. We write $E=\left\{V, G, m, P O I_{m}, A, c\right\}$, where:

- $\quad V$ is the set of visitors, where each visitor $v$ has his own characteristics (i.e. his profile, with e.g. demographic and psychological elements).

- $G=$ is the set of visitor groups, where each group $g$ is defined as the set of visitors belonging to it.

- $\quad m$ is the museum, with its characteristics (i.e. mainly spacial and structural). This is the physical space in which visitors evolve (meaning we have $s=m$ ).

- $P O I_{m}$ is the set of $P O I$ (Point of Interests) of the museum. It includes mainly the exhibits of the museum but potentially other things (e.g. shop, coffee).

- $A$ is the set of IT applications offering specific services to visitors (e.g. in our case, personalised recommendation) and interacting with the Cyber part of our system: e.g., gathering data from sensors or interaction logs to reach a given objective.

- $\quad c$ represents the context elements, in particular gathered from sensors in the museum (e.g. cameras giving the number of persons in a room, noise level indicators, proximity or location sensors, etc.). It brings also constraints external to the museum like regulation or security rules.

According to equation (4), we can write the recommendation problem in a museum as follows, for an application $a \in A$ offering personalised recommendation (e.g. a mobile guide or App.) and a user $u \in U$, where the set of users $U$ is defined as the set of visitors and groups using the application $a, U \subset$ $(V \cup G): \forall u \in U, u \operatorname{ses}(u, a)$ :

$$
P R_{u}^{(\text {museum })}=f\left(u, P O I_{m}, m, c r_{u}, a, c\right),
$$

In this equation, the crowd of the visitors for $u$ is defined as $c r=V \cup G-\{u\}$ if $u$ visits alone, or $c r=V \cup G-\left\{g_{u}\right\}$ when $u$ belongs to a group $g_{u}$ and we assume the group can be considered as a coherent entity, behaving like a single visitor. It is important to consider separately the user and the other visitors, because what is important is their mutual influence.

Let us now define the objectives and constraints linked to each entity. The difference between the two has to be clearly kept in mind: constraints must be respected, while objectives can be partially fulfilled. Typically, constraints induce rules or filters restricting the solution search space of the recommender system, while objectives are formalised with cost functions (also called objective functions) that have to be maximised or minimised.

Lets define $O_{U}$ and $C O_{U}$ as respectively the set of (sets of) objectives and the set of (sets of) constraints of the set of users $U$. We have $O_{U}=\left\{O_{u i}\right\}=$ $\left\{O_{v i}\right\}_{i=1 . . k \leq\|V\|} \cup\left\{O_{g i}\right\}_{i=1 . . l \leq\|G\|}$, and $C O_{U}=\left\{C O_{u i}\right\}=$ 
$\left\{C O_{v i}\right\}_{i=1 . . k \leq\|V\|} \cup\left\{C O_{g i}\right\}_{i=1 . . l \leq\|G\|}$. Visitors have personal interests $I N T_{v}$ (for specific topics or things), as well as constraints $C O_{v}$ and preferences $P_{v}$ (e.g. time constraints, crowd tolerance, distance tolerance, visiting style, see e.g. [7] [8]). Each group can be assimilated to an aggregated visitor having its own interests. Each group comprises individuals having their own interests, but behaving as a single entity, the group. The interests of a group $g$ is a function of the individual interests of its members $v i$ : $I N T_{g}=f\left(I N T_{v 1}, I N T_{v 2}, \ldots, I N T_{v n}\right), v i \in g$. This is a typical scenario of the Social Choice problem, that a group of people arrive at a saddle point where a group-wise decision has to be made that satisfies some notion of consensus among the group. However it does not necessarily corresponds to the best compromise between individual interests. A classical example is a family following the interests of the children of the group. Similarly, constraints and preferences of a group $g$ are defined as a function of the individual constraints and preferences: $C O_{g}=f\left(\left\{C O_{v i}\right\}_{i=1 . . n}\right)$ and $P_{g}=f\left(\left\{P_{v i}\right\}_{i=1 . . n}\right), v i \in g$; Finally, each individual set of objectives is a function of the user constraints, interests and preferences:

$$
O_{u}=f\left(I N T_{u}, P_{u}, C O_{u}\right) .
$$

Postulate 1: $O_{u}$ is fulfilled for a user $u$ when his interests are fulfilled (maximisation), taking into account the given constraints (all) and fulfilling preferences (maximisation).

Each museum has its own constraints $C O_{m}$ and objectives $O_{m}$, which obviously impact the visit: e.g., opening hours, maximum number of people in a room, kind and distribution of exhibits, targeted visitor experience or audience, expected revenues, etc. (see e.g. [7]). This influence the way exhibits are organised, creating potentially predefined paths inside the museums that visitors are supposed to follow to actually have the expected experience. Constraints impacting visits can also be attached to POI like, e.g., maximum crowd capacity or environmental constraints (e.g. light or air flow conditions). Exhibits are organised according to the museum objectives (e.g., following the purpose of an exhibition or according to specific educational purposes), but also potentially other kinds of POIs (e.g. shops, to get some additional revenues from the visits). The objectives of the museum can be related to the expected visitor experience:

$$
O_{m}=f\left(V E x p_{m}\right) \text {. }
$$

We write $O_{A}$, the objectives of IT applications deployed in the museum. Each application $a \in A$ follows its objectives $O_{a}$ that target a specific visitor experience:

$$
O_{a}=f\left(V \operatorname{Exp}_{a}\right) \text {. }
$$

The fulfilment of $V E x p_{m}$ is prepared by structuring the museum and organising exhibitions accordingly. IT applications might be included in this organisation (e.g. including fixed multimedia interactive devices, like computers or TVs). In this case $V E x p_{a}$ is included in $V E x p_{m}$ and $O_{a}$ is coherent with $O_{m}$, meaning that the fulfilment of the museum objectives implies that the application objectives are also fulfilled. However the dynamics introduced by mobile devices make that fulfilling $V E x p_{a}$ might not be possible by a physical reorganisation of the museum. In this case, fulfilling $O_{a}$ can only be realised under the constraints introduced for fulfilling $O_{m}$.
$C O_{c r}$ is the set of objectives linked to the crowd. The fact that a visitor is evolving in a crowd of other visitors induces constraints the personalised application needs to respect. For example, the distribution of the crowd in the museum needs to stay viable for each visitor at any time, meaning that the application should ensure not to drive too many users to a same place or along the same path at the same time.

Last, as said, the context brings constraints such as regulations that have to be applied.

\section{B. Application to a personalised museum guide}

In the case of a mobile personalized recommender system to drive visitors inside a museum, the problem is to compute the recommendations in such a way that: $(\mathrm{O} 1:) o_{A M}$ is fulfilled (the specific objective of the recommendations), (O2:) $O_{V}$ is fulfilled the best possible (knowing (1) is prioritary) so that user experience is personalized, and (O3:) $o_{M}$ is fulfilled the best possible. Considering now that in the way they are defined, all the objectives in $\mathrm{O}$ comprise in fact a set of associated constraints (which we name resp. $c O_{A M}, C O_{V}$ and $c o_{M}$ ), we can define the problem more precisely as: "optimizing $O_{A M}$, while finding the best compromise between $O_{V}$ and $o_{M}$ fulfilment knowing $o_{A M}$, under the constraint that $\mathrm{co}_{A M}$, $C O_{V}$ and ${ }^{C O} M$ must be respected".

As an example, here are some of the objectives and constraints we have in our project (see [8] for more details on the user parameters):

- $o_{A M}$ : historical reflection and reinterpretation;

- $O_{V}$ : personalisation of recommendations;

- $o_{M}$ : leading visitors to paintings not commonly visited, avoid congestion points, etc.;

- $c_{A M}$ : only paintings related to reflection use-cases, specific order between paintings following semantic links, etc.;

- $C O_{V}$ : time constraints, crowd tolerance, etc.;

- $\quad c_{M}$ : structure, opening time, daily painting organisation, etc.

\section{CONCLUSION}

Visitors of cultural heritage sites evolve in a physical environment which by itself brings it's own constraints and also influences the people to behave in certain ways adding to the complexity of the personalization problem. This essentially means providing recommendation and guidance to users tailored to their perceived points of interests (POI), which implies finding the best compromise between the preferences or characteristics of users knowing the site's objectives and guiding them according to the physical constraints. When user groups are involved, this comes additionally to find the best compromise that satisfies some notion of consensus among the group.

The work presented here opens a different perspective on personalisation, introducing the notion of Cyber-Physical and Social System, which is not well known in the research community of user modelling and personalisation. The goal 
of this paper was to make a first step towards extending and formalising the notion of personalisation in the CPSS domain, by taking the particular perspective of personalised recommendations in cultural heritage sites. We formulate the personalization problem as a function of the user, the physical space, the crowd of other persons in the physical space, the IT application implementing personalized services and the global context.

Incorporating the human dimension together with CyberPhysical systems is becoming a recent research trend, understanding it's importance and associated effects, formalized as Cyber-Physical Social systems. The introduction of IT devices and services together with humans in cultural heritage spaces transforms the dedicated sites into CPSS where humans play an important role towards the stability and the functioning of the system since they introduce additional systemic objectives and constraints originating from the uncontrollable and yet not fully understood nature of humans (preference, cognition, behaviour and motivation). We argue here that personalisation could be a solution, providing more control on users while enhancing the User eXperience.

In this position paper, we have discussed the influence of personalisation on the CPSS as well as the impact of the system on personalisation, showing that personalisation is clearly a multi-objective and multi-constraints problem both in virtual and physical spaces. An initial formalisation of personalisation in CPSS has been given, which we instantiated to personalised recommendations in cultural heritage sites.

\section{ACKNOWLEDGMENT}

This work has been partially funded by the European Union's Horizon 2020 research and innovation pro- gramme under grant agreement No 693150, CrossCult project (www.crosscult.eu).

\section{REFERENCES}

[1] CyPhERS FP7 project. (2013) Cyber-physical european roadmap and strategy. [Online]. Available: http://cyphers.eu/sites/default/files/d6.1+2report.pdf

[2] C. G. Cassandras, "Smart cities as cyber-physical social systems," Engineering, vol. 2, no. 2, pp. 156 - 158, 2016.

[3] K. J. Murakami, "Cpss (cyber-physical-social system) initiative-beyond cps (cyber-physical system) for a better future," in Keynote Speech, the First Japan-Egypt Conference on Electronics Communication and Computers JEC-ECC, 2012.

[4] Z. Liu, D. Yang, D. Wen, and W. Zhang, "Cyber-physical-social systems for command and control," IEEE Intelligent Systems, vol. Vol. 26, no. No. 4, pp. pp. 92-96, 2011.

[5] Y. Naudet and I. Lykourentzou, "Personalisation in crowd systems," in 2014 9th International Workshop on Semantic and Social Media Adaptation and Personalization, Nov 2014, pp. 32-37.

[6] M. Foulonneau, V. Grouès, Y. Naudet, and M. Chevalier, Recommender Systems and Diversity: Taking Advantage of the Long Tail and the Diversity of Recommendation Lists. John Wiley \& Sons, Inc., 2014, pp. 71-92.

[7] I. Lykourentzou, X. Claude, Y. Naudet, E. Tobias, A. Antoniou, G. Lepouras, and C. Vassilakis, "Improving museum visitors' quality of experience through intelligent recommendations: A visiting style-based approach." in Workshop Proc. of the 9th Int. Conference on Intelligent Environments, ser. Ambient Intelligence and Smart Environments, J. A. Botía and D. Charitos, Eds., 2013, pp. 507-518.

[8] P.-E. Osche, S. Castagnos, A. Napoli, and Y. Naudet, "Walk the line: Toward an efficient user model for recommendations in museums," in 11th International Workshop on Semantic and Social Media Adaptation and Personalization (SMAP 2016), Thessaloniki, Greece, Oct. 2016, pp. $83-88$. 